Original Research Paper

\title{
Enhancing Astragaloside Production in Astragalus membranaceus through Growth Media and Auxins
}

\author{
${ }^{1 \dagger}$ Yun Ji Park, ${ }^{2 \dagger}$ Jae Kwang Kim and ${ }^{1}$ Sang Un Park \\ ${ }^{I}$ Department of Crop Science, Chungnam National University, \\ 99 Daehak-Ro, Yuseong-Gu, Daejeon 305-764, Republic of Korea \\ ${ }^{2}$ Division of Life Sciences and Convergence Research Center for Insect Vectors, \\ Incheon National University, Incheon 22012, Korea
}

Article history

Received: 30-08-2017

Revised: $18-11-2017$

Accepted: 23-01-2018

$\dagger$ Y.J. Park and J.K. Kim contributed equally to this work.

Corresponding Author:

Sang Un Park

Department of Crop Science, Chungnam National

University, 99 Daehak-Ro,

Yuseong-Gu, Daejeon 305-764,

Republic of Korea

Tel: +82-42-821-5730

Fax: +82-42-822-2631

Email:supark@cnu.ac.kr

\begin{abstract}
The secondary metabolites of triterpenoid saponins, isoflavonoids and polysaccharides are present in Astragalus membranaceus those are biologically active. Astragalosides are a major group of identified compounds that have various pharmacological properties. The present study was considered to enhance astragaloside biosynthesis in hairy root cultures of $A$. membranaceus by using different growth media and concentrations of different auxins. Our findings revealed that Schenk and Hildebrandt (SH) medium responded greatly for the production of the highest dry weight. However, the highest astragaloside production was detected while using $\mathrm{SH}$ medium of its half strength. The growth of hairy roots and accumulation of astragaloside in response to different auxinlike Indole-3-Acetic Acid (IAA), Indole-3-Butyric Acid (IBA) and 1-naphthaleneacetic acid (NAA) were different. Hairy root growth rate was increased by the treatment of IBA and NAA, but was unaffected by IAA. Accumulation of Astragaloside was enhanced in response to the treatment of IBA and NAA. In most cases, treatment with the $0.5 \mathrm{mg} \mathrm{L}{ }^{-1}$ NAA accumulated the highest level of stragaloside in the hairy root of $A$. membranaceus, which might, therefore, be utilized as an ideal mass-scale production system for astragalosides.
\end{abstract}

Keywords: Astragaloside, Astragalus membranaceus, Auxin, Media, Hairy Root Culture

\section{Introduction}

Tissue cultures are generating increasing interesting as potential alternatives for achieving useful and valuable biologically active secondary metabolites (Murthy et al., 2014). For getting higher amount of secondary metabolites and other inevitable biologically active compounds with the shortest possible time and biochemical stability, rapid growth rate, independence from environmental variations and productive capacity of secondary metabolites, hairy root is the very efficient method and can be used in the pharmaceutical and biotechnology industries (Ono and Tian, 2011; Zhou et al., 2011; Talano et al., 2012).

Dried root of Astragalus membranaceus, very usually known as Huang Qi, is broadly used in traditional Chinese medicine (Kwon et al., 2013). Its pharmacological properties have also been proven against several important diseases like antidiabetic, analgesic, diuretic, expectorant, hepatoprotective, immunostimulant, sedative and tonic, (Sinclair, 1998).
Several biologically active constituents are present in $A$. membranaceus roots, such as saponins, isoflavonoids, polysaccharides and astragalosides. Astragalosides belong to the class of cycloartane are principal constituents that are also used as quality control markers of the root (Fig. 1) (Kwon et al., 2013). Particularly, the astragaloside IV has the potentiality to use as a neuroprotective, anti-inflammatory and cardio protective agent and has effects on osteogenesis, angiogenesis and metabolic syndrome (Bian et al., 2011; Luo et al., 2004; $\mathrm{Xu}$ et al., 2008; Zhang et al., 2011a; 2011b; 2005).

Selection of good cell lines, media, use of proper elicitors, supplementation of nutrient and precursor, biotransformation, immobilization as well as permeabilization have been accounted to enhance the accumulation of biomass and secondary metabolites, which is desirable (Murthy et al., 2014). Therefore, this study was undertaken to enhance hairy root growth and astragaloside production of $A$. membranaceus through different media and growth regulator especially auxins. 


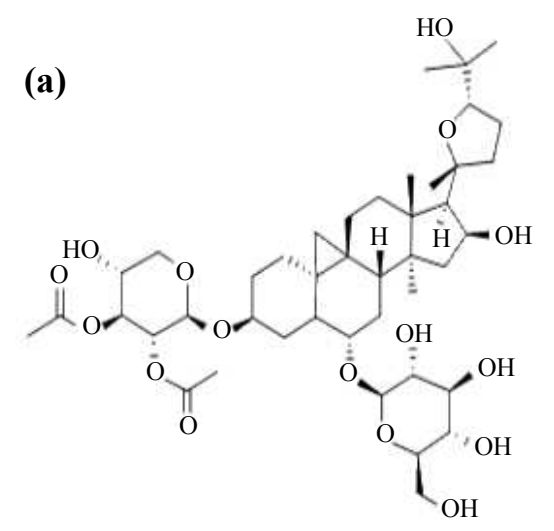

(c)

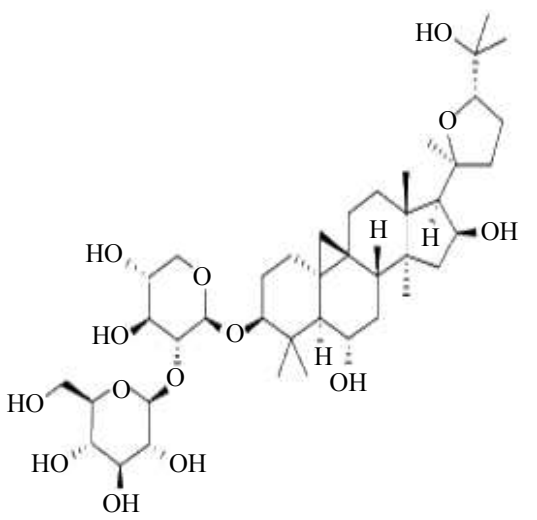

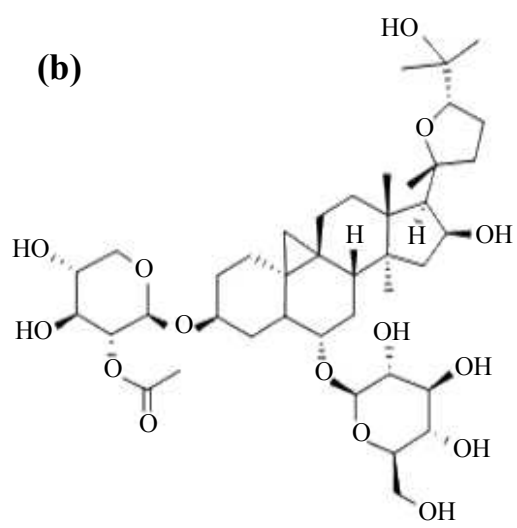

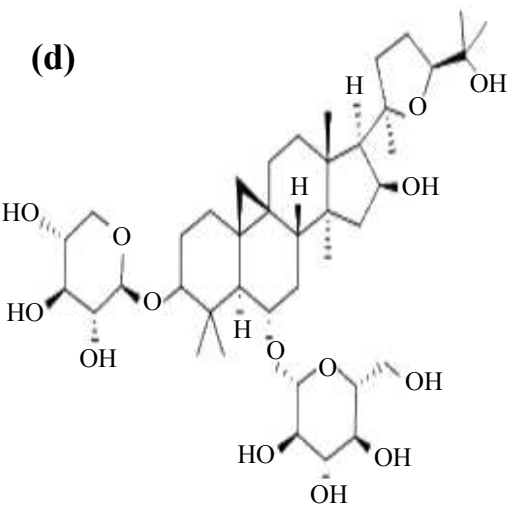

Fig. 1: Structures of astragalosides showing astragaloside I (a), astragaloside II (b), astragaloside III (c) and astragaloside IV (d)

\section{Materials and Methods}

\section{Chemicals}

The astragalosides I, II, III and IV used for external standards were procured from ChromaDex (Irvine, CA, USA). MS basal medium (M4531), SH basal salt mixture (S4828), B5 basal medium (G4410) and IAA (I5897) were obtained from MB Cell (Los Angeles, CA, USA). IBA (1538) was purchased from Phytotechnology Laboratories (Shawnee Mission, KS, USA) and NAA (1465-25-4) was obtained from Kanto Chemical Co (Japan). HPLC-grade methanol was obtained from J. T. Baker Chemical Co., (Phillipsburg, NJ, USA) and formic acid was purchased from Sam Chun Pure Chemical Co., Ltd. (Gyeonggi, Korea).

\section{Hairy Root Cultures Establishment}

A transformed hairy root was initiated from explants of A. membranaceus after being infected with Agrobacterium rhizogenes (strain R1000) according to methods described previously (Fig. 2) (Park et al., 2015). They were subcultured every 20 days in half-strength MS liquid medium having $25^{\circ} \mathrm{C}$ on a shaker $(110 \mathrm{rpm})$ in the dark.

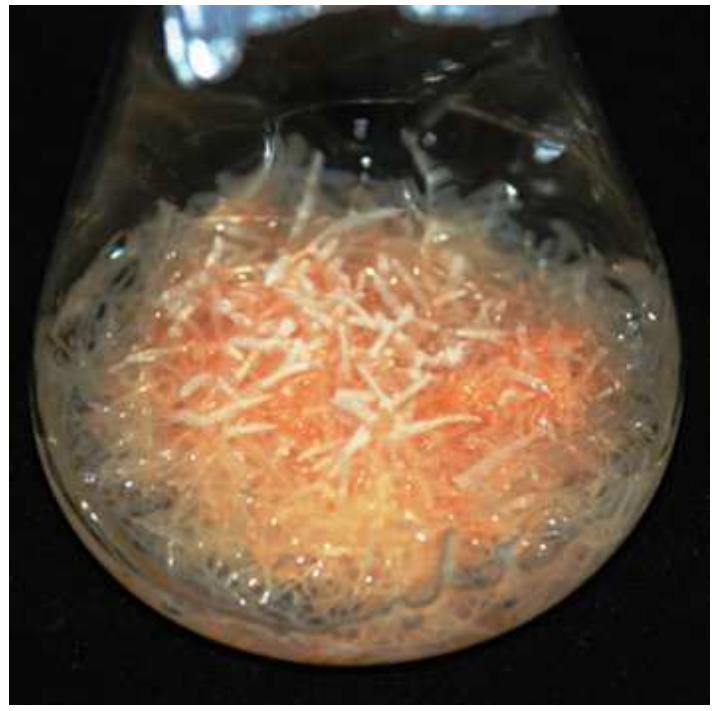

Fig. 2: Photograph of 20-day-old hairy root of $A$. membranaceus grown in SH liquid medium

To determine the optimal medium conditions, $2 \mathrm{~g}$ fresh hairy root segments was inoculated into $30 \mathrm{~mL}$ full- and half-strength MS media (Murashige and Skoog, 
1962), Schenk and Hildebrandt (SH) (Schenk and Hildebrandt, 1972) and Gamborg (B5) (Gamborg et al., 1968). The effect of exogenous auxin treatment was determined in $2 \mathrm{~g}$ of hairy roots cultured on $30 \mathrm{~mL}$ of half-strength MS medium in addition of different concentrations $\left(0.1,0.5\right.$ and $\left.1 \mathrm{mg} \mathrm{L}^{-1}\right)$ of IAA, IBA and NAA. After 20 days, all samples were collected and freeze-dried at $-80^{\circ} \mathrm{C}$ for $72 \mathrm{~h}$.

\section{Extraction of Astragalosides and HPLC Analysis}

Crude extracts from hairy root cultures were obtained by adding $1.5 \mathrm{~mL}$ methanol to $100 \mathrm{mg}$ of the dried sample. The supernatant was filtered through a 0.45- $\mu \mathrm{m}$ Polytetrafluoroethylene (PTFE) syringe filter (Advantec DISMIC-13HP, Toyo Roshi Kaisha, Ltd., Tokyo, Japan) after centrifugation at $4,000 \mathrm{rpm}$ at $25^{\circ} \mathrm{C}$ for $10 \mathrm{~min}$. The astragalosides were divided by using C18 column $(250 \times 4.6 \mathrm{~mm}, 5 \mu \mathrm{m}$; RStech, Daejeon, Korea) through a Futecs model NS-4000 HPLC apparatus (Daejeon, Korea). The drift tube temperature for the Evaporative Light Scattering Detector (ELSD) and nebulizing using a gas flow where rate were set at $70^{\circ} \mathrm{C}$ and $3 \mathrm{~mL} \mathrm{m^{-1 }}$, respectively. formic acid $0.3 \%$ in water (solvent A) and acetonitrile (solvent B) were used as the mobile phase. Samples were eluted with the following gradient: $0 \mathrm{~min}, 75 \% \mathrm{~A} / 25 \% \mathrm{~B} ; 15 \mathrm{~min}, 65 \%$ $\mathrm{A} / 35 \% \mathrm{~B} ; 3 \mathrm{~min}, 55 \% \mathrm{~A} / 45 \% \mathrm{~B} ; 5 \mathrm{~min}, 55 \% \mathrm{~A} / 45 \%$ B; $25 \min , 35 \%$ A/65\% B; 10 min, $20 \%$ A/80\% B; 6 $\min , 20 \% \mathrm{~A} / 80 \% \mathrm{~B}$. The flow rate was retained at 0.8
$\mathrm{mL} \min ^{-1}$. The quantification was done by comparing the test sample retention time to that of a reference and standard curve.

\section{Statistical Analysis}

All data used in this study are the mean values of three independent biological experiments. The statistical analysis was performed using an Analysis of Variance (ANOVA) with Duncan's multiple range test at a level of significance of $\mathrm{p}<0.05$ following the statistical package of SPSS program (SPSS Inc., Chicago, IL, USA).

\section{Results}

\section{Optimization of Hairy Root Culture}

The highest hairy root biomass was produced using SH medium, whereas the lowest was found in B5 medium (Fig. 3). The level of astragalosides was significantly responded by the media type and salt strength (Fig. 4). All types of astragalosides were detected while using $\mathrm{SH}$ and B5 media having their full and half strength. However, using MS media with fulland half-strength contained astragaloside I, II and III, but did not detect any astragalosides IV. Moreover, the levels of astragalosides were higher in the half-strength media than they were in the full-strength media. In most cases, the highest levels of astragalosides were observed in half-strength SH medium.

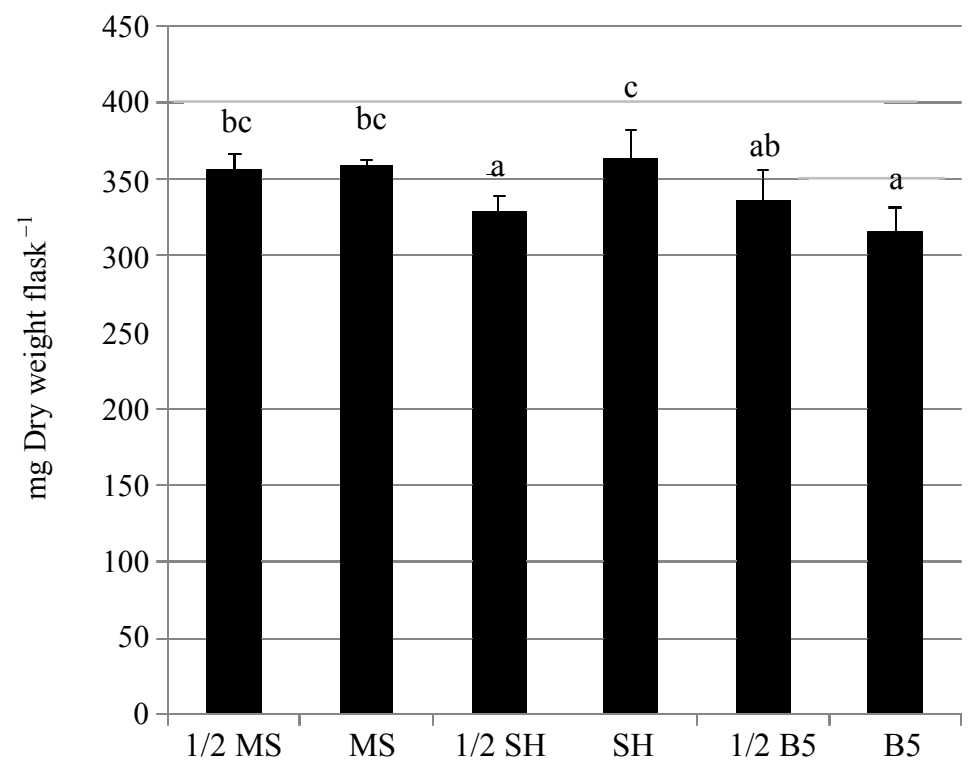

Fig. 3: Effects of different growth media on hairy root biomass in A. membranaceus. Values are means from three independent replicate results \pm standard deviation (SD). In a bar, figures with same letters or without letters do not differ significantly whereas figures with dissimilar letter differ significantly at $\mathrm{p}<0.05$ using DMRT.MS $=$ Murashige and Skoog, $\mathrm{SH}=\mathrm{Schenk}$ and Hildebrandt, B5 = Gamborg 

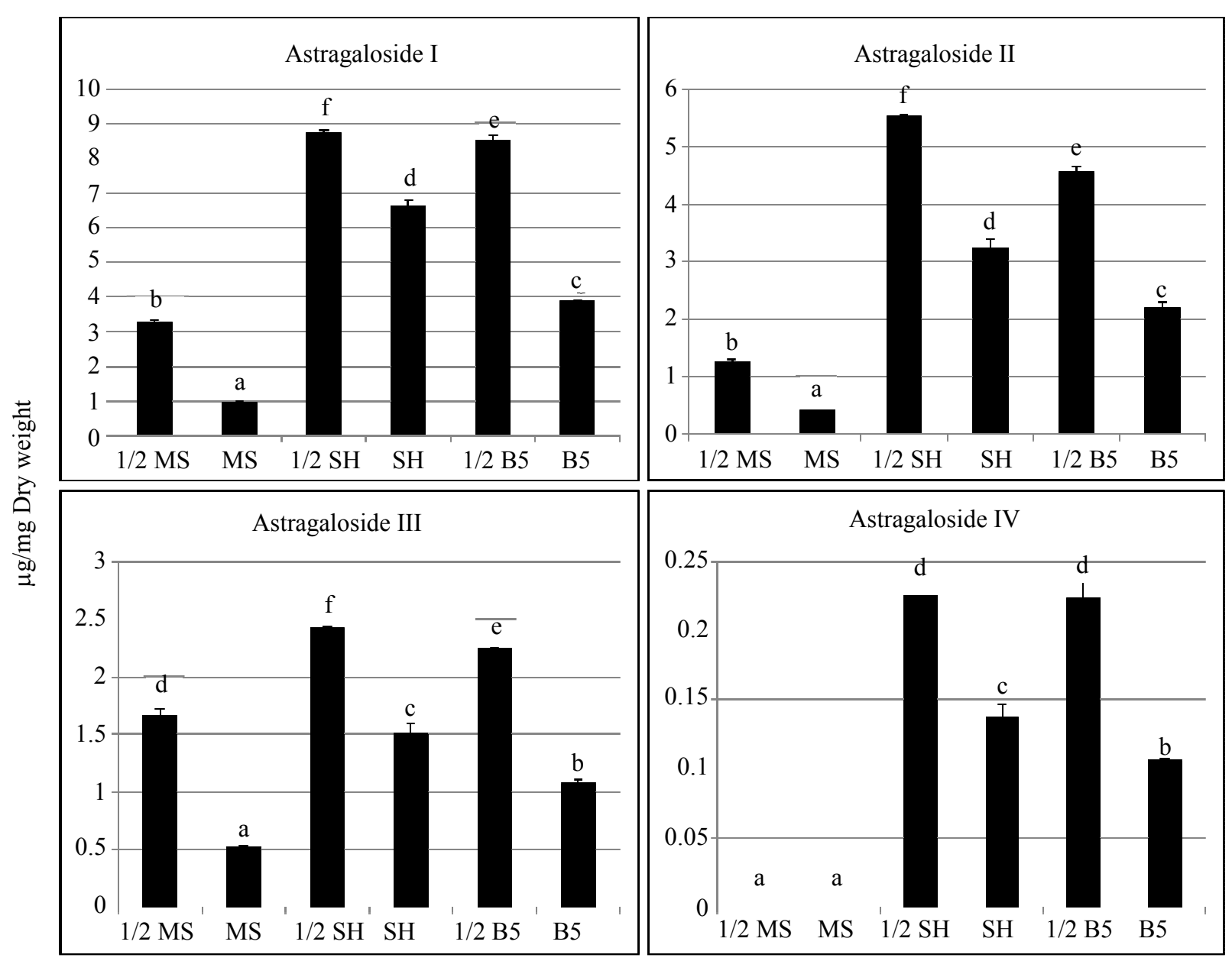

Fig. 4: Astragaloside contents in A. membranaceus hairy root cultures in different media. Values are means from three independent replicate results \pm Standard Deviation (SD). In a bar, figures with same letters or without letters do not differ significantly whereas figures with dissimilar letter differ significantly at $\mathrm{p}<0.05$ using DMRT.MS $=$ Murashige and Skoog, $\mathrm{SH}=\mathrm{Schenk}$ and Hildebrandt, B5 = Gamborg

\section{Effects of Auxin on Growth of Hairy Root and Accumulation of Astragalosides in A. membranacues}

Hairy roots were established using the best growth medium (half-strength MS medium) combined with concentrations $\left(0.1,0.5\right.$ and $\left.1 \mathrm{mg} \mathrm{L}^{-1}\right)$ of IAA, IBA and NAA to investigate the combined response of medium and auxins. Different responses to the auxin were detected in hairy root growth (Fig. 5). Furthermore, IAA treatment showed no positive effect on the growth rate of hairy root. Auxin IBA responded positively in a rapid concentration-dependent for hairy root growth. In particular, MS medium of half strength with $1 \mathrm{mg} \mathrm{L}^{-1}$ IBA produced the maximum biomass productivity. The biomass of the hairy roots was enhanced by NAA at their lower concentrations ( 0.1 and $\left.0.5 \mathrm{mg} \mathrm{L}^{-1}\right)$, but the effect was decreased by high-concentration $\left(1 \mathrm{mg} \mathrm{L}^{-1}\right)$ NAA.
Response of exogenous auxins on astragaloside accumulation in hairy roots of A. membranaceus is shown in Fig. 6. The levels of astragalosides I, II and III decreased at low concentrations of IAA $(0.1$ and $0.5 \mathrm{mg}$ $\mathrm{L}^{-1}$ ), whereas the levels of mentioned astragalosides increased when the concentration was $1 \mathrm{mg} \mathrm{L}^{-1}$ IAA, compared to the control. However, none of the IAA concentrations improved the astragaloside IV production. IBA concentration-dependently increased the content of astragaloside I. In contrast, the synthesis of astragalosides II and III was high in $0.1 \mathrm{mg} \mathrm{L}^{-1}$ of IBA, but declined with increasing concentrations. The astragaloside IV content was detected in hairy roots treated with IBA of 0.5 and $1 \mathrm{mg} \mathrm{L} \mathrm{L}^{-1}$. Among the treatments NAA at $0.5 \mathrm{mg} \mathrm{L}^{-1}$ yielded the highest accumulation of astragalosides II, III and IV. In addition, treatment with NAA at $1 \mathrm{mg} \mathrm{L}^{-1}$ induced the highest formation of astragaloside I. 


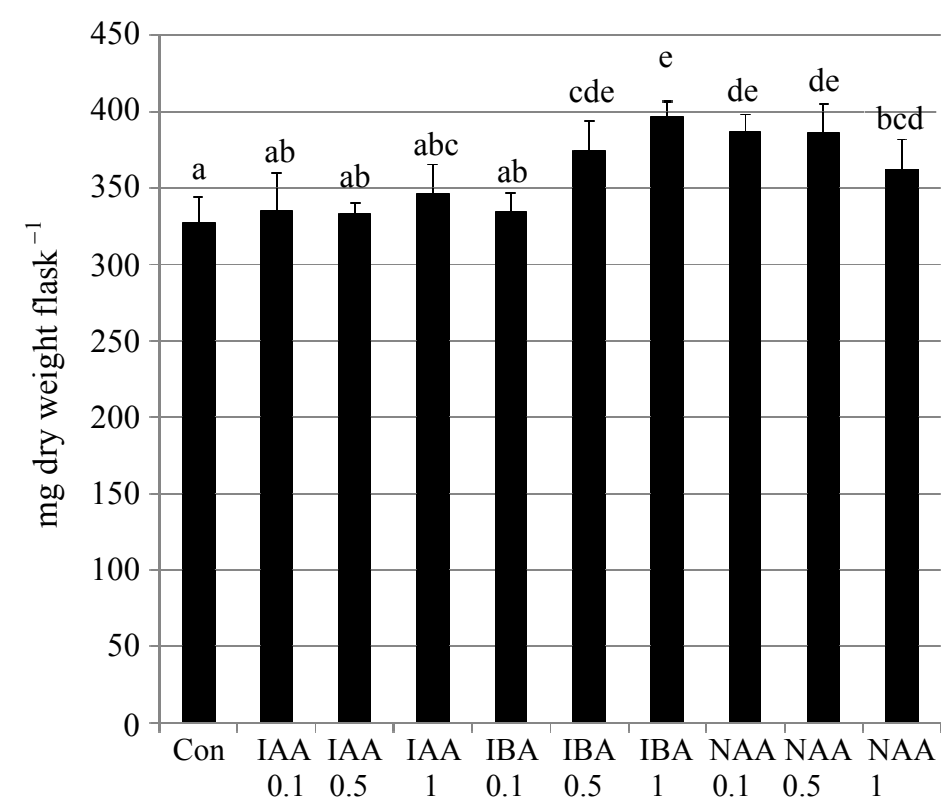

Fig. 5:Effects of auxins on hairy root growth in A. membranaceous. Values are means from three independent replicate results \pm Standard Deviation (SD). In a bar, figures with same letters or without letters do not differ significantly whereas figures with dissimilar letter differ significantly at $\mathrm{p}<0.05$ using DMRT.IAA $=$ Indole-3-Acetic Acid, IBA = Indole-3-Butyric Acid (IBA) and NAA = 1-Naphthaleneacetic Acid

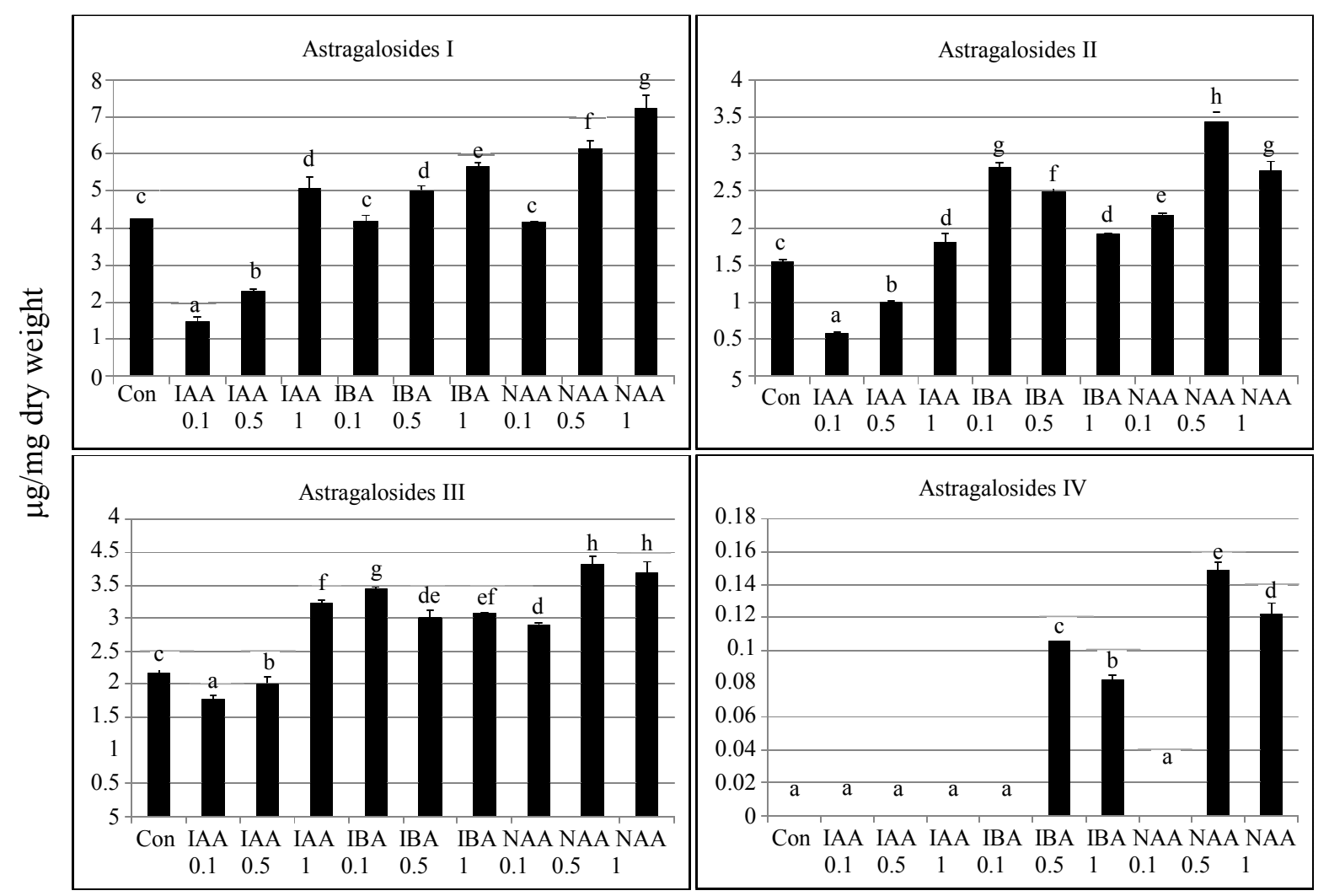

Fig. 6: Effects of exogenous auxins on astragalosides accumulation in hairy root cultures of $A$. membranaceus. Values are means from three independent replicate results \pm standard deviation. In a bar, figures with same letters or without letters do not differ significantly whereas figures with dissimilar letter differ significantly at $p<0.05$ using DMRT.IAA $=$ Indole-3-Acetic Acid, IBA = Indole-3-Butyric Acid (IBA) and NAA = 1-Naphthaleneacetic Acid 


\section{Discussion}

Various factors those could lead to enhance growth and accumulation of different secondary metabolite production are an appropriate type and strength of salt of the medium; elicitors, concentrations of carbohydrates, nitrate and phosphate; and levels of growth regulator. Different medium formulations, including MS, SH, B5 and (LS) (Linsmaier and Skoog, 1965), have been developed and widely used for plant cultures (Murthy et al., 2014). Numerous studies have demonstrated that different media with varying salt strengths affect the accumulation of different compounds and secondary metabolites, in different plant or tissue cultures (Murthy et al., 2014). For instance, Nagella and Murthy (2011) reported that a full-strength MS medium had a significant effect on higher production of biomass and higher level of gymnemic acid accumulation in cell suspension cultures of Gymnema sylvestre. The highest growth rate in the hairy root cultures of ginseng was observed in MS medium having 0.75 -strength, but the level of ginsenoside from a 0.5 -strength MS medium was considerably higher than that from other media (Sivakumar et al., 2005). Therefore, optimization of the medium is crucial for establishing optimal plant cultures.

Exogenous growth regulators have been used to investigate the root biomass and accumulation of secondary metabolites in plant cell, tissue and hairy root cultures (Murthy et al., 2014). Among the various growth regulators, auxins, such as IAA, IBA and NAA play important roles in root development and promoting hairy root induction (Cheruvathur and Thomas, 2014). Moreover, the biomass growth and metabolite accumulation in media treated with different types and concentrations of auxins vary based on plant cell cultures (Mantell and Smith, 1983). Our findings strongly support the results of previous studies that determined the response of exogenous auxin on growth of root biomass and accumulation of natural products in variable plant species, such as Nicotiana tabacum, Morinda citrifolia and Lippia dulcis (Sahai and Shuler, 1984; Sauerwein et al., 1991; Zenk et al., 1975)

\section{Conclusion}

Finally, we investigated the optimal conditions for accumulation of astragaloside in hairy root cultures of A. membranaceus. The findings of this study confirmed that hairy roots grow better in SH medium containing 3\% sucrose and that the half-strength $\mathrm{SH}$ medium was superior to other media for astragaloside production. Moreover, treatment with exogenous auxins, especially NAA, significantly enhanced biomass and astragaloside productivity. Therefore, hairy root culture is an ideal ways for the production of large amounts of biomass with increased astragaloside accumulation for A. membranaceus.

\section{Funding Information}

This work was supported by Korea Institute of Planning and Evaluation for Technology in Food, Agriculture, Forestry and Fisheries (IPET) through Advanced Production Technology Development Program (or Project), funded by Ministry of Agriculture, Food and Rural Affairs (MAFRA) (116115-03-1-CG000).

\section{Author Contributions}

Yun Ji Park: Performed the experiments, analyzed the data and prepare the manuscript.

Jae Kwang Kim: Performed the experiments, analyzed the data and prepare the manuscript.

Sang Un Park: Designed the experiments, coordinated the implementation of research work.

\section{Conflict of Interest}

There is no conflict of interest among the authors.

\section{References}

Bian, Q., J.H. Huang, Q.Q. Liang, B. Shu and W. Hou et al., 2011. The osteogenetic effect of astragaloside IV with centrifugating pressure on the OCT-1 cells. Pharmazie, 66: 63-68.

DOI: $10.1691 /$ ph.2011.0219

Cheruvathur, M.K. and T.D. Thomas, 2014. Effect of plant growth regulators and elicitors on rhinacanthin accumulation in hairy root cultures of Rhinacanthus nasutus (L.) Kurz. Plant Cell Tissue Organ Culture, 118: 169-177. DOI: 10.1007/s1 1240-014-0473-9

Gamborg, O.L.C., R.A. Miller and K. Ojima, 1968. Nutrient requirements of suspension cultures of soybean root cells. Exp. Cell Res., 50: 151-158. DOI: 10.1016/0014-4827(68)90403-5

Kwon, H.J., J. Hwang, S.K. Lee and Y.D. Park, 2013. Astragaloside content in the periderm, cortex and xylem of Astragalus membranaceus root. J. Natural Med., 67: 850-855.

DOI: $10.1007 / \mathrm{s} 11418-013-0741-8$

Linsmaier, E.M. and F. Skoog, 1965. Organic growth factor requirements of tobacco tissue cultures. Physiol. Plantarum, 18: 100-127. DOI: 10.1111/j.1399-3054.1965.tb06874.x

Luo, Y., Z. Qin, Z. Hong, X. Zhang and D. Ding et al., 2004. Astragaloside IV protects against ischemic brain injury in a murine model of transient focal ischemia. Neurosci. Lett., 363: 218-223.

DOI: 10.1016/j.neulet.2004.03.036

Mantell, S.H. and H. Smith, 1983. Cultural factors that influence secondary metabolite accumulations in plant cell and tissue cultures. Seminar series-Society for Experimental Biology. 
Murashige, T. and F. Skoog, 1962. A revised medium for rapid growth and bio assays with tobacco tissue cultures. Physiol. Plantarum, 15: 473-497. DOI: $10.1111 /$ j.1399-3054.1962.tb08052.x

Murthy, H.N., E.J. Lee and K.Y. Paek, 2014. Production of secondary metabolites from cell and organ cultures: strategies and approaches for biomass improvement and metabolite accumulation. Plant Cell Tissue Organ Culture, 118: 1-16.

DOI: $10.1007 /$ s1 1240-014-0467-7

Nagella, P. and H.N. Murthy, 2011. In vitro production of gymnemic acid from cell suspension cultures of Gymnema sylvestre R. Br. Eng. Life Sci., 11: 537-540. DOI: $10.1002 /$ elsc. 201000167

Ono, N.N. and L. Tian, 2011. The multiplicity of hairy root cultures: Prolific possibilities. Plant Sci., 180: 439-446. DOI: 10.1016/j.plantsci.2010.11.012

Park, Y.J., A.A. Thwe, X. Li, Y.J. Kim and J.K. Kim et al., 2015. Triterpene and flavonoid biosynthesis and metabolic profiling of hairy roots, adventitious roots and seedling roots of Astragalus membranaceus. J. Agric. Food Chem., 63: 8862-8869. DOI: $10.1021 /$ acs.jafc.5b02525

Sahai, O. and M. Shuler, 1984. Environmental parameters influencing phenolics production by batch cultures of Nicotiana tabacum. Biotechnol. Bioeng., 26: 111-120. DOI: 10.1002/bit.260260202

Sauerwein, M., T. Yamazaki and K. Shimomura, 1991. Hernandulcin in hairy root cultures of Lippia dulcis. Plant Cell Rep., 9: 579-581. DOI: $10.1007 / \mathrm{BF} 00232336$

Schenk, R.U. and A. Hildebrandt, 1972. Medium and techniques for induction and growth of monocotyledonous and dicotyledonous plant cell cultures. Canad. J. Botany, 50: 199-204.

DOI: $10.1139 / \mathrm{b} 72-026$

Sinclair, S., 1998. Chinese herbs: A clinical review of Astragalus, Ligusticum and Schizandrae. Alternative Med. Rev., 3: 338-344. PMID: 9802911

Sivakumar, G., K. Yuz, J. Hahn and K. Paekz, 2005. Optimization of organic nutrients for ginseng hairy roots production in large-scale bioreactors. Curr. Sci., 89: 641-649.

Talano, M.A., A.L. Oller, P.S. Gonzalez and E. Agostini, 2012. Hairy roots, their multiple applications and recent patents. Recent Patents Biotechnol., 6: 115-133. PMID: 22642821

Xu, X.L., X.J. Chen, H. Ji, P. Li and Y.Y. Bian et al., 2008. Astragaloside IV improved intracellular calcium handling in hypoxia-reoxygenated cardiomyocytes via the sarcoplasmic reticulum $\mathrm{Ca}^{2+}$-ATPase. Pharmacology, 81: 325-332.

DOI: $10.1159 / 000121335$
Zenk, M.H., H. El-Shagi and U. Schulte, 1975. Anthraquinone production by cell suspension cultures of Morinda citrifolia. Planta Med., 28: 79-101. DOI: $10.1055 / \mathrm{s}-0028-1104768$

Zhang, L., Q. Liu, L. Lu, X. Zhao and X. Gao et al., 2011a. Astragaloside IV stimulates angiogenesis and increases hypoxia-inducible factor- $1 \alpha$ accumulation via phosphatidylinositol 3-kinase/Akt pathway. J. Pharmacol. Exp. Therapeut., 338: 485-491.

DOI: $10.1124 /$ jpet.111.180992

Zhang, N., X.H. Wang, S.L. Mao and F. Zhao, 2011 b. Astragaloside IV improves metabolic syndrome and endothelium dysfunction in fructose-fed rats. Molecules, 16: 3896-3907.

DOI: $10.3390 /$ molecules 16053896

Zhang, W., C. Zhang, R. Liu, H. Li and J. Zhang et al., 2005. Quantitative determination of Astragaloside IV, a natural product with cardioprotective activity, in plasma, urine and other biological samples by HPLC coupled with tandem mass spectrometry. J. Chromatography B, 822: 170-177.

DOI: 10.1016/j.jchromb.2005.05.034

Zhou, M.L., X.M. Zhu, J.R. Shao, Y.X. Tang and Y.M. $\mathrm{Wu}, 2011$. Production and metabolic engineering of bioactive substances in plant hairy root culture. Applied Microbiol. Biotechnol., 90: 1229-1239. DOI: $10.1007 / \mathrm{s} 00253-011-3228-0$

\section{Abbreviations \\ ANOVA, Analysis of Variance; B5, Gamborg; ELSD, Evaporative Light Scattering Detector; HPLC, High- Performance Liquid Chromatography; IAA, Indole-3- Acetic Acid; IBA, Indole-3-Butyric Acid; LS, Linsmaier and Skoog; $\mathrm{MeOH}$, Methanol; MS, Murashige and Skoog; NAA, 1-Naphthaleneacetic Acid; PTFE, Polytetrafluoroethylene; SH, Schenk and Hildebrandt; SPSS, Statistical Package for the Social Science.}

